\title{
¿Qué inyectables pueden ser administrados por vía oral o enteral?
}

\author{
Gregorio Romero Candel ${ }^{a}$ y Francisco Tejada Cifuentes ${ }^{b}$.
}

a Residente de Farmacia Hospitalaria. Complejo Hospitalario Universitario de Albacete. Albacete (España).

b Farmacéutico de Atención Primaria. Complejo Hospitalario Universitario de Albacete. Albacete (España).

Dirección para

correspondencia:

Francisco Tejada Cifuentes.

Farmacia de Atención Primaria.

Complejo Hospitalario

Universitario de Albacete.

C/ Hermanos Falcó, 37. C.P. 02006 - Albacete (España).

Correo electrónico:

ftejada@sescam.jccm.es

Recibido el 26 de marzo de 2015.

Aceptado para su publicación el 2 de junio de 2015.

\begin{abstract}
RESUMEN
En este artículo recogemos los principios éticos y legales cuando hemos de decidir sobre el uso de un medicamento en condiciones distintas a las de su ficha técnica. Los medicamentos no siempre están disponibles en formulaciones adecuadas para pacientes con dificultades para tragar o pacientes con sondas de alimentación enteral. Por lo tanto, la modificación de las formas farmacéuticas puede ser necesaria. Se proporciona una lista de inyectables que pueden ser administrados por vía enteral mediante sondas de alimentación o por vía oral cuando no hay alternativas disponibles. Sin embargo, la idoneidad para la administración oral o enteral puede variar ampliamente. En este trabajo no consideramos la vía rectal, como una vía enteral adecuada para la administración de inyectables.
\end{abstract}

Palabras clave: Inyecciones. Administración Oral. Vías de Administración de Medicamentos.

\begin{abstract}
Which injectable medication can be administered orally or enterally?

In this article we collect the ethical and legal principles when we have to decide on the use of a drug in conditions other than those on its data sheet. Medicines are not always available in formulations which are suitable for patients with swallowing difficulties or patients with enteral feeding tubes. The alteration of medication formulations may therefore be necessary. This text provides a list of injectables that can be administered orally or via enteral feeding tubes when there are no available alternatives. However, they vary widely in their suitability for oral or enteral feeding administration. In this paper we do not consider the rectal route, as an adequate enteral route for administration of injectables.
\end{abstract}

Keywords: Injections. Administration, Oral. Drug Administration Routes.

La forma farmacéutica es la disposición individualizada del principio activo y sus excipientes. Estas formas farmacéuticas pueden ser sólidas, semisólidas, líquidas y gaseosas. A su vez los medicamentos tienen diferentes vías de administración, siendo la tecnología farmacéutica la encargada de adecuar la forma farmacéutica y la vía de administración. Pueden existir varias vías de administración para algunas formas farmacéuticas, aunque siempre será la ficha técnica del medicamento donde se recoge la forma farmacéutica y su vía de administración idónea.

En la práctica clínica nos surgen problemas en aquellos pacientes que tiene dificultad para tragar o que son portadores de una sonda nasogástrica, ya que la formulación sólida no es idónea para este tipo de pacientes y la líquida pudiera no estar aprobada para administrar por vía oral. En estos casos, la utilización de otra vía de administración diferente a la aprobada pudiera ser necesaria.

Cuando decidimos administrar una forma farmacéutica por una vía diferente 
a la de su aprobación, ésta queda fuera de la indicación recogida en la ficha técnica del medicamento. Desde este momento, el fabricante ya no es responsable de cualquier evento adverso o fracaso terapéutico que pudiera aparecer durante el tratamiento.

Esta modificación trae consigo implicaciones para los profesionales responsables de la prescripción, dispensación y administración de los medicamentos, ya que esta utilización del medicamento no está avalada, y por tanto será el profesional y no el laboratorio el responsable de cualquier evento adverso que el paciente pudiera experimentar ${ }^{1}$.

Aun cuando esté justificada la administración de un fármaco por una vía diferente a la que está autorizada en la ficha técnica, hay que respetar unos principios legales y éticos que van desde la autonomía, la justicia, el principio de beneficencia, el derecho a la intimidad y la vulnerabilidad y el nivel de dependencia que presentan los pacientes con dificultad para tragar².

Además, debemos ser conscientes de que, por muy seguros que estemos de nuestra decisión, es posible que aparezcan reacciones inesperadas potencialmente graves, por lo que se requiere una vigilancia más estrecha por parte del personal sanitario.

Solo se podrá administrar un fármaco por una vía diferente a la autorizada cuando lo indique el médico responsable. No obstante, dicho procedimiento puede llevarse a cabo por el personal de enfermería, siempre que la orden sea transmitida de manera escrita.

El Real Decreto 1015/2009, de 19 de junio ${ }^{3}$, por el que se regula la disponibilidad de medicamentos en situaciones especiales recoge el acceso a medicamentos en condiciones diferentes a las autorizadas. En el artículo 13 se indica que esta utilización de medicamentos en condiciones diferentes a las autorizadas tendrá carácter excepcional y se limitará a las situaciones en las que se carezca de alternativas terapéuticas autorizadas para un determinado paciente, respetando en todo caso las restricciones que se hayan establecido ligadas a la prescripción o dispensación del medicamento y el protocolo terapéutico asistencial del centro sanitario. En el artículo 15 se recogen las obligaciones del médico responsable del tratamiento. Estas obligaciones son:
- Informar al paciente, en términos comprensibles, de la naturaleza del tratamiento, su importancia, implicaciones y riesgos, y obtener su consentimiento conforme a la Ley 41/2002, de 14 de noviembre.

- Notificar las sospechas de reacciones adversas de acuerdo a lo previsto en el Real Decreto 1344/2007, de 11 de octubre.

- Respetar en su caso las restricciones que se hayan establecido ligadas a la prescripción o dispensación y el protocolo terapéutico asistencial del centro sanitario.

Uno de los objetivos de la bioética ${ }^{4}$, en lo que respecta a la relación asistencial con el enfermo, cualquiera que sea su patología, será la búsqueda de soluciones concretas a casos clínicos concretos, siempre que se originen conflictos, lo cual ocurre con mucha frecuencia en la situación de enfermedad incapacitante o terminal. La bioética entrará en acción cuando aparezca la posibilidad de elegir entre varias opciones, que a veces podrán ser completamente contradictorias. Ante la falta de una certeza absoluta, el dilema consistirá en buscar la forma más adecuada para tomar la mejor decisión, es decir, cómo diferenciar lo correcto de lo incorrecto, lo que es bueno de lo que es malo o incluso cómo escoger la mejor opción entre varias opciones correctas.

La bioética entra en juego en situaciones especialmente delicadas como la sedación terminal, y aunque la administración de un medicamento por una vía diferente a la autorizada no es ni de lejos comparable, es positivo el hecho de que cada vez que un profesional se plantee la cuestión de cómo actuar en estos casos, reflexione, a ser posible en colaboración con el enfermo, su familia y el resto del equipo asistencial, sobre la actitud a tomar y se trate el problema de manera individualizada. Lo habitual es tener dudas en la toma de decisiones ante esta situación, por lo que la prudencia será la mejor consejera, y resultaría pretencioso creerse en posesión de la certeza absoluta.

Siempre deberemos tener en cuenta las condiciones del principio ético del doble efecto ${ }^{5,6}$. En este caso es difícil de llevar a la práctica, de modo que se puede utilizar de forma reducida e incorrecta, valorando únicamente la intención de la persona que realiza la acción. Se tiende a resolver la cuestión de la siguiente forma: "como la intención es buena, se puede realizar la acción" o "como la intención declarada es buena, se considera que la acción es correcta". 
Existen unos preceptos legales y actuaciones reguladas relacionadas con estas intervenciones que los profesionales sanitarios debemos conocer:

- Ley $41 / 2002$, de 14 de noviembre, reguladora de la autonomía del paciente y de derechos y obligaciones en materia de información y documentación clínica. Arts. 4, 5, 8 y 9.

- Información sobre la adopción de la medida: La autorización de la medida excepcional y urgente deberá ser comunicada al paciente de modo adecuado a sus posibilidades de comprensión y a las circunstancias existentes en el momento. También debe informarse a las personas que, vinculadas a él por razones familiares o de hecho, se encuentren acompañando al paciente. La información debe darse con carácter previo, durante el mantenimiento y posteriormente.

- Historia clínica y documentos del protocolo: Debe quedar constancia en la historia clínica de las circunstancias que han justificado tanto la adopción de esta medida como su mantenimiento en el tiempo

Para cada medicamento debemos elegir la forma farmacéutica adecuada y realizar una preparación y administración correcta para no obstruir la sonda, conseguir su efectividad, y evitar efectos adversos e interacciones.

En la tabla 1 se recogen diferentes principios activos cuyos preparados inyectables se pueden administrar por vía oral o sonda gástrica, y las recomendaciones para su uso. Aunque la rectal es una vía de administración enteral y que proporciona una rápida absorción de los principios activos, no la hemos considerado en este texto debido a la complejidad que tiene la administración de fármacos que no han sido específicamente diseñados para esta vía.

\begin{tabular}{|c|c|}
\hline Fármaco & Instrucciones \\
\hline Acetazolamida & $\begin{array}{l}\text { El inyectable reconstituido puede ser administrado por vía enteral }{ }^{1,2} \text {. Puede ser almacenado } \\
\text { en frigorífico durante } 24 \text { horas }^{1} \text {. }\end{array}$ \\
\hline Acetilcisteína & $\begin{array}{l}\text { La solución inyectable diluida a } 50 \mathrm{mg} / \mathrm{ml} \text { puede ser administrada por vía enteral }{ }^{1,2} \text {. Tiene un } \\
\text { sabor muy amargo }{ }^{1,2} \text {. El jarabe de naranja o de grosella negra }{ }^{2,7} \text {, zumo de naranja o refres- } \\
\text { cos de cola pueden ser utilizados para diluir la solución inyectable }{ }^{2,7,8} \text {. }\end{array}$ \\
\hline Aminocaproico, ácido & Administrar directamente. Se puede administrar conjuntamente con la nutrición enteral ${ }^{9}$. \\
\hline Aminofilina & $\begin{array}{l}\text { La inyección se ha utilizado por vía enteral. Como se trata de una preparación de liberación } \\
\text { inmediata, son necesarios ajustes, como dividir la dosis diaria en 3-4 dosis separadas. }\end{array}$ \\
\hline Arginina & El inyectable puede ser administrado por vía enteral ${ }^{2,7}$. \\
\hline Atropina & El inyectable puede ser administrado por vía enteral ${ }^{2,8}$. \\
\hline Benztropina & $\begin{array}{l}\text { El inyectable puede ser administrado por vía oral }{ }^{2} \text {. También ha sido administrado vía sonda } \\
\text { gástrica, aunque el fabricante no recomienda esta vía }{ }^{2} \text {. }\end{array}$ \\
\hline Butilescopolamina & Administrar directamente. Se puede administrar conjuntamente con la nutrición enteral ${ }^{9}$. \\
\hline Calcio folinato & El inyectable puede ser administrado por vía oral ${ }^{1,2}$. \\
\hline Cimetidina & $\begin{array}{l}\text { El inyectable puede ser administrado por vía enteral }{ }^{1,2} \text {. La absorción de cimetidina puede } \\
\text { verse reducida cuando se administra directamente en el yeyuno }{ }^{2} \text {. }\end{array}$ \\
\hline Clonacepam & $\begin{array}{l}\text { El inyectable puede ser administrado por vía enteral }{ }^{2,7} \text { después de ser diluido con } 1 \mathrm{ml} \text { de } \\
\text { agua de inyección }{ }^{2} \text { (importante tener en cuenta los excipientes: etanol, ácido acético, alco- } \\
\text { hol bencílico y propilenglicol) })^{2,7} \text {. }\end{array}$ \\
\hline Clonidina & $\begin{array}{l}\text { El inyectable puede ser administrado por vía enteral }{ }^{1,2} \text {. Se puede administrar directamente o } \\
\text { bien diluir con agua para conseguir un volumen adecuado }{ }^{2} \text {. El inyectable es insípido, pero si } \\
\text { se desea se puede mezclar con zumo de fruta en el momento de la administración }{ }^{2} \text {. }\end{array}$ \\
\hline Clorfeniramina & El inyectable puede ser administrado por vía enteral². \\
\hline Clorpromacina & Administrar directamente. No se debe administrar conjuntamente con la nutrición enteral ${ }^{9}$. \\
\hline Ciclicina & $\begin{array}{l}\text { El inyectable se puede administrar por vía enteral; sin embargo los fabricantes no tienen } \\
\text { información suficiente al respecto, y por lo tanto no lo recomiendan². }\end{array}$ \\
\hline
\end{tabular}




\begin{tabular}{|c|c|}
\hline Ciclofosfamida & $\begin{array}{l}\text { El inyectable puede ser usado directamente vía sonda gástrica, o bien ser usado para pre- } \\
\text { parar una solución por vía oral }{ }^{1} \text {. }\end{array}$ \\
\hline Deferoxamina & $\begin{array}{l}\text { El inyectable puede ser administrado por vía oral o por sonda nasogástrica en un volumen } \\
\text { de } 50-100 \mathrm{ml} \text { de agua }{ }^{2} \text {. Tiene un sabor desagradable }{ }^{2} \text {. }\end{array}$ \\
\hline Dexametasona & El inyectable puede ser administrado por vía enteral ${ }^{2,8}$. \\
\hline Diacepam & $\begin{array}{l}\text { El inyectable se ha administrado por vía enteral; sin embargo, los fabricantes no tienen infor- } \\
\text { mación suficiente al respecto y, por lo tanto no lo recomiendan². Se puede producir pérdida } \\
\text { de principio activo cuando se administra a través de tubos de PVC relativamente largos, ya } \\
\text { que el diacepam se puede adsorber especialmente en las paredes del tubo (portex-PVC). El } \\
\text { diacepam también puede contribuir a la obstrucción de los tubos }{ }^{2} \text {. }\end{array}$ \\
\hline Digoxina & $\begin{array}{l}\text { La inyección se ha dado por vía enteral en algunos centros, pero no es recomendable por } \\
\text { su biodisponibilidad impredecible }{ }^{2} \text {. }\end{array}$ \\
\hline Dinoprostona & El inyectable se debe diluir con agua para su administración por vía enteral2,7,8. \\
\hline Dipiridamol & $\begin{array}{l}\text { El inyectable puede ser administrado por vía oral o por sonda }{ }^{1,2} \text {. Dipiridamol se debe ad- } \\
\text { ministrar con el estómago vacío, por lo que la alimentación enteral se debe suspender una } \\
\text { hora antes y reanudar una hora después de la administración del fármaco }{ }^{2} \text {. }\end{array}$ \\
\hline Disopiramida & $\begin{array}{l}\text { El inyectable debe ser administrado por vía enteral. Es muy amargo y tiene un efecto anes- } \\
\text { tésico a nivel local en la boca, por lo que debe administrarse con mucho cuidado por vía } \\
\text { oral }^{2} \text {. }\end{array}$ \\
\hline Fitomenadiona & $\begin{array}{l}\text { Está autorizado para uso por vía oral (pero no para uso por sondal) })^{2,7} \text {. Se recomienda enjua- } \\
\text { gar cuidadosamente antes y después la sonda si es administrado por esta vía². }\end{array}$ \\
\hline Flecainida & $\begin{array}{l}\text { El inyectable se debe administrar directamente sin diluir por vía enteral. Solo se debe utilizar } \\
\text { en situaciones de emergencia, y el paciente debe ser monitorizado para evitar la aparición } \\
\text { de efectos clínicos adversos. Si se administra por sonda de alimentación enteral, siempre } \\
\text { se debe enjuagar con agua desionizada, y no se debe mezclar con soluciones alcalinas, ni } \\
\text { con iones sulfato, fosfato o cloruro. No mezclar este fármaco con otros previamente a su } \\
\text { administración }{ }^{2} \text {. }\end{array}$ \\
\hline Furosemida & Diluir en $50 \mathrm{ml}$ de agua la ampolla de $20 \mathrm{mg} / 2 \mathrm{ml}^{9}$. \\
\hline Glicopirronio & La solución inyectable se ha utilizado por vía enteral2,7. \\
\hline Hidralacina & El inyectable reconstituido puede ser administrado por vía enteral ${ }^{1,2,7}$. \\
\hline Hidrocortisona &  \\
\hline Hioscina (butilbromuro) & $\begin{array}{l}\text { El inyectable puede ser administrado por vía enteral }{ }^{1,2,7} \text {. El contenido de la ampolla puede } \\
\text { ser almacenado en un refrigerador hasta } 24 \text { horas una vez abierta la misma }{ }^{2} \text {. }\end{array}$ \\
\hline Hioscina (hidrobromuro) & $\begin{array}{l}\text { El inyectable debería ser administrado por vía oral }{ }^{2,7} \text {, aunque también ha sido usado por } \\
\text { sonda }^{2} \text {. }\end{array}$ \\
\hline Ketamina & $\begin{array}{l}\text { El inyectable diluido puede ser administrado por vía oral. Debe ser mezclado con una bebi- } \\
\text { da aromatizada para enmascarar el sabor amargo }{ }^{2} \text {. }\end{array}$ \\
\hline Labetalol & $\begin{array}{l}\text { El inyectable debe ser administrado por vía oral con zumo de frutas para evitar el sabor } \\
\text { amargo }{ }^{1,2,7} \text {. También se podría administrar por sonda }{ }^{2} \text {. }\end{array}$ \\
\hline Levomepromacina & $\begin{array}{l}\text { El inyectable puede ser administrado por vía oral }{ }^{1} \text {. También ha sido administrado por } \\
\text { sonda }{ }^{2} \text {. Debe tenerse en cuenta que los excipientes se pueden degradar a productos que } \\
\text { teóricamente pueden inducir ataques de asma cuando se administra por vía enteral. No } \\
\text { existen datos de ataques de asma registrados por el laboratorio, por lo que el riesgo se } \\
\text { puede considerar bajo². }\end{array}$ \\
\hline Medroxiprogesterona & Ha sido administrado oralmente, aunque existen pocos datos que respalden el uso ${ }^{2}$. \\
\hline Metadona & Administrar directamente. Se puede administrar conjuntamente con la nutrición enteral. \\
\hline Metamizol & Diluir en $20 \mathrm{ml}$ de agua la ampolla de $2 \mathrm{mg} / 5 \mathrm{ml}^{9}$. \\
\hline
\end{tabular}




\begin{tabular}{|c|c|}
\hline Metotrexato & $\begin{array}{l}\text { El inyectable puede ser diluido con agua y ser administrado por vía oral. La fecha de caduci- } \\
\text { dad puede ser ampliada si se utiliza el conservante adecuado'. La absorción del inyectable } \\
\text { alcanza una concentración plasmática similar a la obtenida con los comprimidos }{ }^{1} \text {. }\end{array}$ \\
\hline $\begin{array}{l}\text { Metilprednisolona (suc- } \\
\text { cinato sódico) }\end{array}$ & $\begin{array}{l}\text { Este inyectable se ha administrado por vía enteral }{ }^{8,10} \text {, aunque el fabricante no recomienda su } \\
\text { uso por esta vía. }\end{array}$ \\
\hline Metoclopramida & El inyectable ha sido administrado por vía enteral ${ }^{2}$. \\
\hline Metoprolol & $\begin{array}{l}\text { La inyección se ha administrado por vía enteral en algunos centros, pero hay muy poca } \\
\text { información sobre el tema, por lo que no es recomendable }{ }^{2} \text {. }\end{array}$ \\
\hline Midazolam & $\begin{array}{l}\text { La inyección puede ser administrada por vía oral. La inyección tiene un sabor amargo que } \\
\text { se puede diluir con zumo de manzana, jarabe de frambuesa o cereza, chocolate o refrescos } \\
\text { de cola } a^{2,7} \text {. }\end{array}$ \\
\hline Micofenolato mofetilo & $\begin{array}{l}\text { El inyectable reconstituido puede ser administrado por vía enteral, con un lavado previo y } \\
\text { posterior de la sonda con una solución de dextrosa al } 5 \% \text {. Debe extremarse la precaución } \\
\text { al manipular el polvo del vial (riesgo teratogénico). En caso de contaminación lavar inmedia- } \\
\text { tamente con agua y jabón (en caso de contaminación ocular lavar con abundante agua) }{ }^{2} \text {. }\end{array}$ \\
\hline Ondansetrón & $\begin{array}{l}\text { El inyectable ha sido usado por vía enteral. Es preferible que la administración por sonda } \\
\text { sea directamente en el yeyuno, cuando el jarabe de la mezcla contenga sorbitol. El inyecta- } \\
\text { ble tiene carácter ácido, por lo que se recomienda enjuagar la sonda antes y después de la } \\
\text { administración para evitar la precipitación del fármaco }{ }^{2} \text {. La mezcla es estable en zumo de } \\
\text { manzana }^{2} \text {. }\end{array}$ \\
\hline Pentazocina & $\begin{array}{l}\text { El inyectable mezclado con zumo de naranja inmediatamente antes de su administración } \\
\text { puede ser administrado vía enteral }{ }^{2} \text {. }\end{array}$ \\
\hline Pentoxifilina & Administrar directamente. Se puede administrar conjuntamente con la nutrición enteral ${ }^{9}$. \\
\hline Petidina & El inyectable puede ser administrado vía enteral ${ }^{2}$. \\
\hline Piracetam & Administrar directamente. Se puede administrar conjuntamente con la nutrición enteral ${ }^{9}$. \\
\hline Propanolol & $\begin{array}{l}\text { El inyectable ha sido administrado por vía enteral; si se administra por vía oral debería ser } \\
\text { mezclado con jarabe de frambuesa. De todos modos existe poca información al respecto, } \\
\text { por lo que debería ser evitado². }\end{array}$ \\
\hline Ranitidina & El inyectable puede ser administrado por vía enteral ${ }^{1,2}$. \\
\hline Sodio (bicarbonato) & El inyectable puede ser administrado por vía enteral ${ }^{2}$. \\
\hline Sodio (cloruro) & El inyectable puede ser administrado por vía enteral². \\
\hline Sodio (fenilbutirato) & El inyectable puede ser administrado por vía oral². \\
\hline Teofilina & Administrar directamente. No se debe administrar conjuntamente con la nutrición enteral ${ }^{9}$. \\
\hline Tranexámico, ácido & $\begin{array}{l}\text { El inyectable puede ser administrado por vía oral }{ }^{1} \text { o por sonda inmediatamente después } \\
\text { de su disolución }{ }^{2} \text {. No debe mantenerse la ampolla más de } 24 \text { horas en el frigorífico tras su } \\
\text { apertura }{ }^{1} \text {. }\end{array}$ \\
\hline Vancomicina & $\begin{array}{l}\text { El inyectable está autorizado para administración vía oral o por sonda para el tratamiento de } \\
\text { enterocolitis estafilocócica o colitis pseudomembranosa por Clostridium difficile. Después } \\
\text { de la reconstitución, la dosis seleccionada debe ser diluida en } 30 \mathrm{ml} \text { de agua } a^{1,2,11-13} \text {. Es } \\
\text { aconsejable la mezcla de la solución con jarabes aromatizantes en el momento de la admi- } \\
\text { nistración }{ }^{2,11-13} \text {. La caducidad del inyectable reconstituido depende de las recomendaciones } \\
\text { de la marca }{ }^{2} \text {. Las indicaciones para la vancomicina inyectable y la vancomicina enteral son } \\
\text { diferentes. No debe intercambiarse la ruta de administración sin consultar con un especia- } \\
\text { lista en el tema }{ }^{2} \text {. }\end{array}$ \\
\hline Verapamilo & $\begin{array}{l}\text { El inyectable puede ser administrado por vía enteral }{ }^{1,2,7} \text {. Administrar con el estómago vacío; } \\
\text { suspender la alimentación enteral durante una hora antes y una hora después de cada } \\
\text { dosis }^{2} \text {. }\end{array}$ \\
\hline
\end{tabular}

Tabla 1. Relación de principios activos cuyos preparados inyectables pueden ser administrados por vía enteral, y recomendaciones de uso. 


\section{Aspectos a tener en cuenta:}

- Este texto no proporciona una lista completa de los inyectables que se puede administrar por vía enteral.

- La administración de inyectables por vía enteral por lo general da lugar a una práctica no autorizada. La responsabilidad legal de los profesionales de la salud no se considera en este documento.

- Este documento solo tiene en cuenta la administración enteral de los inyectables en los pacientes con dificultades para tragar 0 pacientes con sondas de alimentación enteral. Otras preparaciones, que pudieran ser más adecuadas, no se han considerado.

- Este documento proporciona información general sobre principios activos y por lo tanto, las diferencias entre las marcas no son consideradas.

- Este documento no tiene en cuenta las interacciones entre los fármacos y el material plástico de los tubos de nutrición enteral.

- La posible alteración de los perfiles farmacocinéticos cuando los medicamentos se administran en una forma no autorizada no ha sido considerada.

Por lo tanto, es importante tener en cuenta que cuando tengamos un paciente que requiere medicación oral y es portador de una sonda de alimentación gástrica o bien presenta dificultades para tragar, podemos considerar utilizar vías diferentes de las que el medicamento tiene autorizadas $^{2}$. Únicamente, cuando no dispongamos de alternativas por vía oral, se administrarán estos medicamentos por una vía no autorizada en la ficha técnica.

Previamente a administrar los inyectables a través de las sondas de alimentación enteral o por vía oral debemos valorar su idoneidad, ya que la administración enteral presenta una amplia variabilidad inter e intraindividual ${ }^{1}$.

Para disminuir en lo posible esta variabilidad, debemos tener en cuenta distintos factores que pueden modificar la eficacia y seguridad del fármaco, como son: situación de la sonda, forma farmacéutica empleada o interacciones con la nutrición enteral y con el material de la sonda.

\section{BIBLIOGRAFÍA}

1. White R, Bradnam V. Drug Administration via Enteral Feeding Tubes [Internet]. Londres: Royal Pharmaceutical Society; 2011 [acceso 04/05/2011]. Disponible en: http:// www.medicinescomplete.com.

2. Smyth J. The NEWT Guidelines [Internet]. North Wales: Betsi Cadwaladr University Local Health Board (East): 2013 [acceso 02/08/2013]. Disponible en: http://www. newtguidelines.com/

3. Real Decreto $1015 / 2009$, de 19 de junio, por el que se regula la disponibilidad de medicamentos en situaciones especiales. Boletín Oficial del Estado, núm 174 (20/07/2009).

4. Azulay A. La sedación terminal. Aspectos éticos. An Med Interna 2003; 20 (12): 645-9.

5. Quill TE, Dresser R, Brock DW. The rule of double effect - a critique of its role in end-of-life decision making. $\mathrm{N}$ Eng $\mathrm{J}$ Med 1997; 337 (24): 1768-71.

6. Sulmasy DP, Pellegrino ED. The rule of double effect: clearing up the double talk. Arch Intern Med. 1999; 159 (6): 545-50.

7. Paediatric Formulary Committee. BNF for Children [Internet]. Londres: BMJ Group, Pharmaceutical Press, and RCPCH Publications; 2013 [acceso 07/08/2013]. Disponible en: http://www.medicinescomplete.com

8. Martin J, editor. BNF for Children 2011-12 [Internet]. Londres: British Medical Association, the Royal Pharmaceutical Society of Great Britain, the Royal College of Paediatrics and Child Health, and the Neonatal and Paediatric Pharmacists Group [acceso 16/09/2013]. Disponible en: http://bnfc.org/bnfc/

9. Guía de administración de fármacos por sonda. Versión 1.0, junio 2008. Servicio de Atención Farmacéutica Especializada de Badajoz. Gerencia del Área de Salud de Badajoz.

10. Antal EJ, Wright CE 3rd, Gillespie WR, Albert KS. Influence of route of administration on the pharmacokinetics of methylprednisolone. J Pharmacokinet Biopharm. 1983; 11 (6): 561-76.

11. Electronic Medicine Compendium (eMC). Summary of Product Characteristics - Vancomycin [Internet]. Wockhardt UK Ltd. Date of last authorisation 20/05/2008 [acceso 13/05/2014]. Disponible en: http://www.emc. medicines.org.uk.

12. Electronic Medicine Compendium (eMC). Summary of Product Characteristics - Vancomycin [Internet]. Hospira UK Ltd. Date of revision of the text 30/01/2009 [acceso 13/05/2011]. Disponible en: http://www.emc.medcicines. org.uk.

13. Electronic Medicine Compendium (eMC). Summary of Product Characteristics - Vancocin [Internet]. Flynn Pharma Ltd. Date of revision of the text 10/11/2008 [acceso 13/05/2011]. Disponible en: http://www.emc.medicines. org.uk. 